REVISTA DE DERECHO UNED, NÚM. 11, 2012

\title{
CONSIDERACIONES CASUÍSTICAS ACERCA DE LA NOCIÓN DE COMBUSTIBLE EN LA JURISPRUDENCIA ROMANA
}

\section{CONSIDERATIONS ABOUT THE CONCEPT OF FUEL IN ROMAN JURISPRUDENCE}

\section{Marta-Natalia LóPez Gálvez}

Profesora Dra. Colaboradora del Departamento de Derecho Romano Facultad de Derecho. U.N.E.D.

Resumen: En el presente trabajo se parte del examen de la etimología del término combustible y de su precedente semántico en Roma. Se analiza su noción en relación con el concepto de leña a propósito del contenido de un legado de este carácter, y se pretende determinar qué se consideró como leña para quemar y cuáles fueron sus utilidades en el marco de la casuística jurisprudencial, desde los llamados veteres en tiempos de la república hasta la disposición de la noción de leña bajo el título 'De verborum significatione' del Digesto de Justiniano. Con ello se examinan los criterios que fueron progresivamente usados para delimitar el concepto jurídico de leña, y se trazan posibles semejanzas con otros términos de cuño moderno como el de biomasa.

Abstract: This paper is part with the examination of the etymology the term fuel and its semantic precedent in Rome. It is mainly dicussed fuel about the content of a legate of wood, and also it is intended to determine what was considered a fuel object -with the utility wich presents- in the scope of casuistry jurisprudence since veteres from the republican times until the moment this notion is settled under the title 'De verborum significatione' in the Digest of Justinian. It is studied the criteria that were being used increasingly 
to define the legal concept, and drawing possible similarities with other modern coined terms as the biomass.

Palabras clave: Combustible, Legado, Lignum, Materia, Biomasa, Digestorum Similitudines.

Keywords: Fuel, Legacy, Lignum, Materia, Biomass, Digestorum Similitudines.

Recepción original: 25/10/2012.

Aceptación original: 29/10/2012.

Sumario: I. Introducción. Consideraciones sobre la etimología y semántica del término; II. Elaboración jurisprudencial romana sobre la cuestión; II.1. Consideraciones generales; II.2. Los veteres; II.3. Jurisprudencia del Principado; II.4. Jurisprudencia clásica tardía; II.4.A. El pensamiento de Ulpiano; II.4.A.a) El comentario ulpianeo a Mucio Scevola a propósito de la diferencia entre leña y madera; II.4.A.b) El comentario ulpianeo a Ofilio sobre aquello que sólo se puede aprovechar para hacer fuego y la denominación de leña para hacer carbón; II.4.A.c) Otras inclusiones al concepto de leña; II.4.A.d) La leña en el legado de un fundo; II.4.B. Pomponio y Paulo; II.5. Reelaboradores postclásicos y justinianeos. El Libro 50,16 de Digesto De verborum significatione; III. Tradición jurisprudencial y otras conclusiones.

\section{INTRODUCCIÓN. CONSIDERACIONES SOBRE LA ETIMOLO- GÍA Y SEMÁNTICA DEL TÉRMINO}

El objeto del presente estudio es delimitar en el Derecho Romano el precedente del término combustible, tanto en relación a su etimología, significado, como en la determinación de qué pudo considerarse objeto de combustible, y señalar posibles similitudes y diferencias con el término actual, y con algunas de las modalidades en que éste puede presentarse.

Por ello iniciamos este trabajo haciendo referencia a la etimología y significado del término del que partimos -combustible-, que recogido en la vigésima segunda edición del Diccionario de la Real Academia Española ${ }^{1}$-D.R.A.E.-, señala «combusto» como el origen latino de la voz y cuya semántica responde a las siguientes acepciones, $1^{\text {a }}$ que puede arder, $2^{\mathrm{a}}$ que arde con facilidad, $3^{\mathrm{a}}$, leña, carbón, petróleo que se usa en las cocinas, chimeneas, hornos, fraguas y

\footnotetext{
${ }^{1}$ Real Academia Española, Diccionario de la Lengua Española, Madrid 1992, vol.1.
} 
máquinas cuyo agente es el fuego. Por otra parte, en nuestros días, el combustible puede presentar múltiples formas, reconociéndose como una de ellas el denominado «biocombustible ${ }^{2}$ definido como aquél producido directa o indirectamente a partir de la biomasa ${ }^{3}$, entendiéndose por ésta última la materia de origen biológico ${ }^{4}$. Una clasificación básica entre los biocombustibles es aquella que distingue entre los de carácter primario y secundario, según se usen esencialmente en su forma natural ó sea precisa la elaboración. Entre los primeros se encuentran la leña -junto a las astillas y gránulos de madera-, cuya combustión directa se usa para satisfacer la necesidad de combustible para cocinar o generar calor. Entre los segundos figura el carbón vegetal -reiteramos a modo de producto elaborado $-^{5}$. Con carácter general hoy en día se considera que pueden extraerse biocombustibles sólidos, líquidos, y gaseosos de los derivados forestales, de los cultivos, y de los desechos, como son los desechos agrícolas -en los cultivos-, o animales -estiércol-, entre otros ${ }^{6}$. Así pues, se ha considerado que la biomasa cuya combustión produce energía se obtiene fundamentalmente por tres procesos ${ }^{7}$ :

- Estableciendo determinados cultivos que pueden transformarse posteriormente en combustible.

- Aprovechando los residuos forestales, agrícolas, domésticos, que pueden ser transformados en combustibles (biomasa residual).

- Mediante la transformación química o biológica de ciertas especies vegetales por ejemplo en carbón.

Teniendo en cuenta lo anterior, llama la atención que el vocablo combustum haya podido utilizarse con la misma forma linguística y básicamente el mismo significado por diversos juristas del periodo

${ }^{2}$ El término ha sido definido en la «Terminología Unificada sobre la Bioenergía» (que será citada como TUB), en una publicación de la Food and Agriculture Organization -en adelante FAO- en diciembre de 2004, y adoptada asimismo por la Agencia Internacional de la Energía -AIE-, vid. http://www.fao.org/bioenergy/52184/es/\#b .

${ }^{3}$ Tomado este concepto de biomasa del documento TUB de la FAO.

${ }^{4}$ Se excluye la encastrada en yacimientos y formaciones geológicas fosilizadas.

${ }^{5}$ FAO, El estado mundial de la agricultura y alimentación. Panorama técnico, Roma 2008.

${ }^{6}$ Como son los cultivos propiamente energéticos, residuos de agroindustria, de industria alimentaria y del papel, residuos sólidos urbanos, fangos cloacales y el biogás proveniente de la digestión de residuos agrícolas u otros residuos orgánicos.

7 FORUM ATÓMICO ESPAÑOL, El libro de la Energía. Madrid 1992; Cuestiones sobre la Energía. Madrid 1993. VIAN ORTUÑO, A., Química Industrial, Barcelona, editorial Reverte, 1994. 
clásico, comenzando por los veteres - de la primera etapa clásica-, como es el caso de Mucio Scevola, hasta Ulpiano, jurista clásico tardío. Así es, Ulpiano en un comentario a Sabino en el libro 25, Ulp. $25 \mathrm{ad} S \mathrm{Sab}$. recogido en Digesto 32,55 y a propósito de tratar sobre lo que ha de entenderse por leña «lignum» ${ }^{8}$ en un legado testamentario, hace referencia a varias opiniones jurisprudenciales, en las cuales tanto Mucio Scevola, Ofilio, y el propio Ulpiano citan diversas variantes del término combustum, así comburendum -D. 32,55, 1comburendi-D.32, 55pr. y 3-, comburitur-D. 32,55,5- en el sentido de determinar qué material puede considerarse objeto de leña y por tanto servir para hacer fuego, con su consecuente utilidad.

En particular es Ulpiano, Ulp. 25 ad Sab. D.32,55,3, el que asocia la acepción de comburum, con la de combustible en sentido propio y actual, ya que habla de la finalidad de la leña, como aquella dispuesta para quemar, señalando las posibles utilidades de este proceso, entre las que cita el uso para la calefacción de los baños, o de las calderas de las viviendas, bien para los hornos de cal -a modo de pequeña industria- o bien para cocer cualquier otra cosa ${ }^{9}$.

Lignis autem legatis quod comburendi causa paratum est continetur, sive ad balnei calefactionem sive diaetarum hypocaustarum sive ad calcem vel ad aliam rem coquendam solebat uti.

Una vez que se ha mostrado cómo la voz «combustible» encuentra en el Derecho Romano su raíz etimológica y semántica, veamos ahora, principalmente en un debate diacrónico objeto del comentario de Ulpiano pero también en otros textos de Digesto cómo a efectos de determinar el objeto de un legado testamentario de leña ${ }^{10}$ -lignum - se hace referencia a qué materiales pueden ser apropiados como combustible para hacer fuego y rendir a las diversas utilidades o necesidades de la población de Roma.

${ }^{8}$ DAREMBERG-SAGLIO, Dictionnaire des Antiquités Grecques et Romaines, voz Lignum, París, Hachette, 1877, pág. 1243; ROBY, H.J., Roman Private Law in the times of Cicero and of the Antonines, Cambridge, University Press, 1902, reimpresión de 2000, pág. 343, donde se trata del posible contenido de los legados.

${ }^{9}$ Para las traducciones de los textos de este trabajo nos apoyamos en la obra de D'ORS, A., et alii., El Digesto de Justiniano, vol. I, II, y III, Pamplona, ed. Aranzadi, 1968. El texto no figura como objeto de ninguna manipulación posterior, LEVY-RAVEL, Index Interpolationum quae in Iustiniani Digestis inesse dicuntur, Weimar 1929, -en adelante Index Interpolationum - y en la Palingenesia de Lenel, está ubicado bajo el título XXV De legatis, rúbrica De ligno legato, col 1.106, n² 2679, cfr. LENEL, O., Palingenesia Iuris Civilis, reimpr. Graz 1960.

${ }^{10}$ Acerca del legado testamentario en Roma, vid. BIONDI, B., Voz Legato -Diritto Romano-, en Novissimo Digesto Italiano, Torino 1957, vol. 9, pág. 597; VOCI, Voz Legato, P., en Enciclopedia del Diritto, Italia 1973, vol. 23, pág. 709. 


\section{ELABORACIÓN JURISPRUDENCIAL ROMANA SOBRE LA CUESTIÓN}

\section{II.1. Consideraciones generales}

Comenzando con el análisis de las opiniones de la jurisprudencia romana las ordenaremos y trataremos considerando los diversos estratos casuísticos propuestos en la tesis de García Garrido que diferencia entre la elaboración de los veteres; Labeón a Salvio Juliano; juristas de la última etapa clásica, y reelaboradores postclásicos y justinianeos ${ }^{11}$. Partimos de dos respuestas contenidas en Digesto que presentan similitud en su tenor, detectada por la obra Digestorum Similitudines de García Garrido y de Reinoso Barbero ${ }^{12}$ con el número de 5.165. En principio dada la inscriptio los dos textos son de la autoría del jurista clásico tardío Ulpiano. En el texto -A- Ulpiano cita el pensamiento de Ofilio jurisconsulto romano que pertenece al estrato mas antiguo, los veteres. El texto -B- también de Ulpiano se ha incluido en el título 16 del libro 50 de Digesto -intitulado $D e$

${ }^{11}$ GARCÍA-GARRIDO, M.J., Casuismo y jurisprudencia romana, Responsa. Madrid, ed. Ediciones Académicas, 2007, pág. 28, donde se explica la metodología de estudio de los casos ordenados por estratos casuísticos jurisprudenciales diferenciados. Estos estratos son «Estrato A» o jurisprudencia republicana también conocidos como veteres; «Estrato B» o jurisprudencia del Principado, referidos a los juristas de la llamada etapa central o alta, desde Labeón a Salvio Juliano; «Estrato C» o juristas de la última etapa clásica; «Estrato D o reelaboradores postclásicos y compiladores justinianeos». El mismo autor en estudios recientes como Nuevas reflexiones sobre los estratos casuísticos, publicado en Atti dell'Accademia Romanistica Constantiniana, Convegno Internazionale in onore di Giuliano Criffò, y en Miscelánea Romanística vol. III, -en prensa- ed. Universidad Nacional de Educación a Distancia, Madrid, además de citar nuevamente dicha metodología propone designar los estratos por las grandes figuras de la jurisprudencia romana -si bien cuando estos estudios queden finalizados- .

${ }^{12}$ Coincidencias textuales que en su acepción amplia pueden comprender también los denominados textos geminados que se explican con mas detalle infra con ocasión del estudio particular de los dos textos paralelos. Vid. GARCÍA-GARRIDO, M.J., y REINOSO-BARBERO, F., Digestorum Similitudines, vol. VII, Similitud 5.165, Madrid 1994. Para el conocimiento y aplicación de esta obra también puede consultarse GARCÍA GARRIDO, M.J. Digestorum Similitudines, en Miscelánea Romanísitica vol. I, Madrid, ed. Universidad Nacional de Educación a Distancia 2006, pág. 429; Redacciones coincidentes (Leges geminatae) y casos jurisprudenciales semejantes (Capita similia) en Estudios d'Ors, Pamplona 1987, pág. 517 y ss. y en Miscelánea Romanística vol. I cit., pág. 327 Madrid; Similitudines e Masse Bluhmiane, en Orbis Iuris Romani, Bratislava 1988, y en Miscelánea Romanística vol. I, cit., pág. 383; Oratio pro Opera «Digestorum Similitudines» en coautoría con el Prof. Fernández de Marcos, Miscelánea Romanística vol. III, en prensa, cit.; Tratando de fragmentos que presentan similitud vid. también CHIAZZESE, Confronti testuali. Contributo alla dottrina delle interpolazioni gisustinianee, en Annali Palermo 6 (1931) 65 y ss.; REINOSO-BARBERO, F., Siete prevenciones en la interpretación del lenguaje jurisprudencial, en Il Linguaggio dei Giuristi romani 5 (1999) pág. 83-112. 
verborum significatione ${ }^{13}$ - . Así pues, la referencia a Ofilio en el texto -A- y el hecho de que se incluyese la opinio de Ulpiano en el libro De verborum significatione, D.50,16, probablemente por los compiladores justinianeos, va a marcar el arco histórico en relación a una cuestión ¿qué debe entenderse por leña -lignum-?

\section{TEXTO A}

\section{D.32,55,7 Ulp. 25 ad Sabinum ${ }^{14}$}

'Si lignum sit paratum ad carbones coquendas atque conficiendas, ait Ofilius libro quinto iuris partiti carbonum appellatione huiusmodi materiam non contineri: sed an lignorum? et fortassis quis dicet nec lignorum: non enim lignorum gratia haec testator habuit. sed et titiones et alia ligna cocta ne fumum faciant utrum ligno an carboni an suo generi adnumerabimus? et magis est, ut proprium genus habeatur.'

\section{TEXTO B}

\section{D.50,16,167 Ulp. 25 ad Sabinum}

“ Carbonum» appellatione materiam ${ }^{15}$ non contineri: sed an «lignorum?» et fortassis quis dicet nec lignorum: non enim lignorum gratia $^{16}$ habuit. sed et titiones et alia ligna cocta ne fumum faciant utrum ligno an carboni an suo generi adnumerabimus? et magis est, ut proprium genus habeatur. sulpurata quoque de ligno aeque eandem habebunt definitionem. ad faces quoque parata non erunt lignorum appellatione comprehensa, nisi haec fuit voluntas. idem et de nucleis olivarum, sed et de balanis est, vel si qui alii nuclei ${ }^{17}$. de pinu autem integri strobili ligni appellatione continebuntur.'

Pertenecen ambos pasajes a un supuesto en el que aparece un testador cuya voluntad es dejar un legado de leña. La cláusula

${ }^{13}$ En D.50,16, los compiladores manifestaron mediante una selección de términos la importancia de las palabras y de su significado jurídico. Entre otros se han ocupado de esta temática DELL'ORO, A., "Il linguaggio dei compilatori del Digesto quale risulta dal titolo 50.16 "De verborum significatione», Scientia iuris e linguaggio nel sistema giuridico romano, Milano 2001; MARRONE, M., "Le significationes di D. 50,16 («De verborum significatione»)», en SDHI 60 (1994).

${ }^{14}$ Mommsen ya detecta en nt. 28 que los fragmentos de D. 32, 55, 7 a 9 se encuentran repeticiones en D. 50,16,167. Vid. Mommsen-Krueger, Corpus Iuris Civilis, Hildesheim 1993 en nt. 28 al texto.

${ }^{15}$ En texto Mommsen -en adelante Momm-. referencia a nota 5 que remite como ley geminada al primer inciso del texto contenido en D. 32, 55,7. En adelante ref. a nt.

${ }_{16}$ Momm. ref. a nt. 6 que remite como ley geminada a D. 32, 55,7.

${ }^{17}$ Momm. ref. a nt. 6 que remite como ley geminada a D. 32, 55,7. 
testamentaria debió suscitar un conflicto entre los herederos y el legatario. Los herederos defenderían un concepto restrictivo y el beneficiado con el legado esgrimía un concepto amplio. A raíz de esta controversia se expresan las respuestas diversas de los jurisconsultos, que como hemos mencionado comenzarán con los veteres y finalizarán con la inclusión del texto en el libro De verborum significatione de Digesto. La similitud entre ambas respuestas y el estudio de los textos que la componen se examina -infra-, ya que a este punto del trabajo sólo pretendemos que ofrezca el marco histórico para el análisis del concepto de leña, a través de las distintas etapas de elaboración jurisprudencial.

\section{II.2. Los veteres}

Perteneciente al primer estrato casuístico -A-, e incluso con anterioridad a Ofilio, en los albores del S. I adC., Mucio Scevola, en su libro 2 de iure civilis ${ }^{18}$, D.32,55pr. ya comienza discerniendo qué es lo que debe entenderse como materia para quemar, para hacer fuego. En este sentido escribe -D.32,55,2- que si se lega un fundo para leña, no ha de considerarse como tal los árboles aún no cortados del mismo, a no ser que estuvieran destinados a ser partidos en trozos pequeños, si bien hay que tener en cuenta que esta última precisión podría haber sido añadida al texto por compiladores posteriore ${ }^{19}$. Así pues, en opinión del jurista, en el legado de un fundo con fin utilizarlo para leña, contrariamente a lo que a priori, podría pensarse, ha de excluirse como tal los árboles que aún no se encuentran cortados, Idem ${ }^{20}$ libro secundo negat arbores nondum concisas, nisi quae minutatim conciduntur, videri ei legatas, cui ligna legata sunt.

Pero aún más, precisa el jurisconsulto, D.32,55pr. que incluso en el caso de los árboles ya cortados habría que distinguir entre la finalidad dada a los mismos, bien previstos para convertirlos en leña o en madera, porque señala Scevola que en el caso de que ya estuvieran cortados no debe entenderse como leña aquellos previstos para el fin de obtener madera -materia- para la construcción.

18 Año 95 adC.

${ }^{19}$ LEVY-RAVEL, Index Interpolationum, cit. donde se señala que los términos nondum minutatim podrían haber sido añadidos posteriormente. Asimismo se consideran sospechosos de interpolación nisi-conciduntur y desde nisi aliud-fin. La ubicación palingnenética de los textos de los veteres se cita a propósito del comentario de Ulpiano a los mismos, tratado posteriormente.

$20 \mathrm{O}$ como señala Momm. ref. a nt. 21 al text: id est Mucius. 
'et Quintus Mucius libro secundo refert, si cui ligna legata essent, quae in fundo erant, arbores quidem materiae causa succisas non deberi: nec adiecit, si non ${ }^{21}$ comburendi gratia succisae sunt, ad eum pertinere, sed sic intellegi consequens est.'

Tratando en particular de materia, décadas después -50 adC.Servio, citado por Pomponio en su libro 30 de comentarios a Sabino, Pomp. 30 ad Sab, D.32,57 en un caso donde ha de determinarse qué ha de contenerse como legado de madera, respondía que no pertenece al legatario de toda la madera, ni el arca ni el armario 'Servius respondit, cui omnis materia legata sit, ei nec arcam nec armarium legatum esse.' Bien podría decirse que la respuesta de Servio no representa valor en este examen de la cuestión dado que a priori en la misma, no se trata en concreto de lignum, pero a estos efectos llamamos la atención sobre el hecho de que al encontrarse la misma ubicada en Digesto a continuación del comentario ulpianeo sobre el significado de lignum y a posteriori de la respuesta de Paulo a Sabino diferenciando entre madera y leña, significa que sirvió a los compiladores para interpretar su contenido a la luz de lo dispuesto en D.32,55, esto es, que sí contribuye por exclusión a la descripción del concepto de lignum al ocuparse de delimitar qué ha de entenderse por madera a propósito de un legado.

Ofilio, en su libro 5 iure partito, D.32,55,1, parece concretar con mayor detalle qué ha de entenderse comprendido en la noción de lignum, señalando que al legatario de leña le pertenece todo lo que no recibe otro nombre, como las varas, el carbón vegetal, y los huesos de aceitunas u orujo ${ }^{22}$.

'Ofilius quoque libro quinto iuris partiti ita scripsit, cui ligna legata sunt, ad eum omnia ligna pertinere, quae alio nomine non appellantur, veluti virgae carbones nuclei olivarum, quibus ad nullam aliam rem nisi ad comburendum possit uti ...'

Por otra parte, en D.32,55,4, Ofilio niega que formen parte de tal concepto de leña los sarmientos, salvo que fuera por expresa voluntas testatoris. Posiblemente tiene presente que en una sociedad eminentemente rural y de economía agrícola los sarmientos presentaban mayor utilidad para fines de agricultura.

Ofilius libro quinto iuris partiti scripsit nec sarmenta ligni appellatione contineri ...

21 Momm. nt. 18 propone también sed conficiendi.

22 D'ORS et alii, El Digesto de Justiniano, cit. para traducir nuclei olivarum utiliza tanto las expresiones de huesos de aceitunas y de orujo, cada una en un texto. 
La última respuesta que se examina de Ofilio citado por Ulpiano, Ulp. 25 ad Sab D.32,55,7 es aquella con la cual en el presente trabajo iniciábamos el primer estrato jurisprudencial, y que plantea, a su vez, si debe entenderse como lignum la leña dispuesta para arder y hacer carbón. Lo que afirma es que si hay leña dispuesta para arder y hacer carbón, no ha de comprenderse en el nombre de carbones ${ }^{23}$.

${ }^{24}$ Si lignum sit paratum ad carbones coquendas atque conficiendas, ait Ofilius libro quinto iuris partiti carbonum appellatione huiusmodi materiam non contineri: ....'

\section{II.3. Jurisprudencia del Principado}

Respecto a este estrato -B- que comprende el periodo entre Labeón y Salvio Juliano, cabe señalar que es precisamente por razón de sus comentarios a Sabino -jurista del Principado- por lo que los juristas clásico tardíos Pomponio (D.32,57), Ulpiano (D.32,55), y Paulo $(D .32,56)$ se ocupan de delimitar qué es objeto de leña o madera a efectos de un legado. Los libros de Sabino que se citan son respectivamente el trigésimo, el vigésimo quinto, y el cuarto.

\section{II.4. Jurisprudencia clásica tardía}

\section{II.4.A. El pensamiento de Ulpiano}

De la jurisprudencia clásica tardía contamos con las respuestas de Pomponio, Ulpiano, y Paulo. Pero el comentario ulpianeo es el más extenso y de él nos ocupamos en mayor medida. Ulpiano, en su libro 25 de comentarios a Sabino, con su espíritu enciclopedista refiere las opiniones de aquellos que le preceden, parafraseando y añadiendo su propio parecer. Así pues, en este epígrafe nos referimos en primer lugar a los comentarios que el jurista realiza a las respuestas de Mucio Scevola y Ofilio para posteriormente exponer otras opiniones del jurista sobre diversas cuestiones.

II.4.A.a) El comentario ulpianeo a Mucio Scevola a propósito de la diferencia entre leña y madera

23 LEVY-RAVEL, Index Interpolationum, cit., donde se indica que el término carbones podría no haber estado incluido en el texto original, desprendiéndose la alusión al mismo sólo por el sentido del texto.

${ }^{24}$ Momm. ref. a nt. 28 donde se indica su similitude con D.50,16,167. 
Ulpiano escribe que si bien la designación de leña es de carácter general, ha de precisarse entre lo que es madera y lo que es leña, y definiendo la leña como lo que se dispone para quemar, y la madera para construir o reparar se plantea la cuestión de si presenta alguna relevancia el hecho de que esté o no partida o cortada. D. 32,55pr. 'Ligni appellatio ${ }^{25}$ nomen generale est, sed sic separatur, ut sit aliquid materia, aliquid ${ }^{26}$ lignum. materia est, quae ad aedificandum fulciendum necessaria est, lignum, quidquid conburendi causa paratum est. sed utrum ita demum, si concisum sit an et si non sit?'

Por ello acude y cita la mencionada opinión de Mucio Scevola en el libro 2 de iure civilis, expuesta en el epígrafe anterior, de la que Ulpiano extrae dos consecuencias. La primera surge ante la afirmación muciana, de que los árboles cortados destinados a construcción -para madera- no han de considerarse leña, Ulpiano parece extraer como regla que los árboles cortados designados para quemar si serían leña. En cuanto a la segunda, Mucio Scevola niega que sean leña los árboles aún no cortados a no ser que hayan sido destinados a esa función, de ahí, Ulpiano deduce que un bosque no puede ser considerado en su conjunto como leña aunque sí considera la excepción de los árboles no destinados en principio a ser leña pero que han sido abatidos.

II.4.A.b) El comentario ulpianeo a Ofilio sobre aquello que sólo se puede aprovechar para hacer fuego y la denominación de leña para hacer carbón

Ulpiano suscribe el pensamiento de Ofilio, D. 32,55,1, en cuanto a que en el concepto de leña entra todo aquello que sólo se pueda aprovechar para hacer fuego, es decir todos los residuos forestales y agrarios que no tengan ninguna otra posible aplicación y que se puedan quemar. Y amplía la relación de posibles residuos que entren en la categoría ya que en el último inciso del texto añade el fruto del quercus (encinas y otros) esto es, balani -bellotas ${ }^{27}$ y otros huesos vegetales

${ }^{25}$ Momm. ref. nt. 16: apellatio. Este mismo término, apellatio, LEVI-RAVEL, Index Interpolationum, cit., también lo considera un añadido posterior al texto original, quizá en la necesidad de una mayor sistemática en la denominación de las cosas. Por otra parte aliquid también se considera sospechoso, y en la Palingenesia de Lenel, está ubicado bajo el título XXV De legatis, rúbrica De ligno legato, col 1.106, nº 2679, cfr. LENEL, O., Palingenesia Iuris Civilis.

${ }^{26}$ Momm. ref. nt. 17: aliud.

${ }^{27}$ Estrabón, Geographica, vol. III, en sus escritos sobre los pueblos del norte de la Península Ibérica señala que durante dos tercios del año, se alimentaban de bellotas de encina, dejándolas secar, triturándolas y fabricando con ellas un pan que se conserva un tiempo. 
'.... sed et balani vel si qui alii nuclei'. Otros ejemplos de residuos agrarios y forestales son citados por el jurista en D.32,55,4 in fine.

A propósito de la cita de Ofilio sobre los sarmientos, Ulpiano, escribe, D.32,55,4, 'Ofilius libro quinto iuris partiti scripsit nec sarmenta ligni appellatione contineri: sed si voluntas non refragatur, et virgulae et gremia ${ }^{28}$ et sarmenta et superamenta materiarum et vitium stirpes atque radices continebuntur ${ }^{29}$. Existe un aparente ius controversum entre la afirmación de Ofilio en la primera parte del texto y la que figura en el último inciso. En la primera se asegura que los sarmientos no son leña, sin embargo en la segunda parte del párrafo Ulpiano incluye a los sarmenta como posible objeto de leña si a ello no se opone la voluntad del testador. No hay tal controversia si interpretamos que en el último inciso se está hablando de los sarmientos a modo de residuo de los mismos, lo que también ocurre con el resto de la relación de derivados forestales con la que finaliza el párrafo como son las varas pequeñas, matorrales, virutas, troncos y raíces de vides.

Otra respuesta de Ofilio que Ulpiano utiliza para clarificar algunos conceptos, Ulp. 25 ad Sab, es la contenida en D. 32,55,730 donde el primero señala que si hay leña dispuesta para arder y hacer carbón, no se comprende en el nombre de carbones, pero se plantea Ulpiano si entonces ha de considerarse como leña $i$.... sed an lignorum? Parece continuar el texto con el comentario de éste escribiendo que es posible que alguno diga que tampoco es leña, pues no lo tenía el testador como tal. En este sentido, nuevamente se pregunta si los tizones y otra leña quemadas para que ardan sin humo han de contarse como leña o como una clase aparte '... sed et titiones et alia ligna cocta ne fumum faciant utrum ligno an carboni an suo generi adnumerabimus? para finalmente considerar en el texto que es mejor tenerlo como clase aparte.

Ulp. 25 ad Sab. D. 32,55,7 'Si lignum sit paratum ad carbones coquendas atque conficiendas, ait Ofilius libro quinto iuris partiti carbonum appellatione huiusmodi materiam non contineri: sed an lignorum? et fortassis quis dicet nec lignorum: non enim lignorum gratia haec testator habuit. sed et titiones et alia ligna cocta

${ }^{28}$ Momm. ref. nt. 23 cremia.

${ }^{29}$ LEVY-RAVEL, Index Interpolationum, cit., Donatuti, plantea si la referencia a la voluntad del testador fue original [si-refragatur]. y en la Palingenesia de Lenel, está ubicado bajo el título XXV De legatis, rúbrica De ligno legato, col 1.106, n 2679, cfr. LENEL, O., Palingenesia Iuris Civilis.

${ }^{30}$ LEVY-RAVEL, Index Interpolationum, cit., en el que se cita como Beseler sugiere como interpolado el tenor entre sed-fin. Además Lenel sospecha de los términos carbonum et lignorum. 
ne fumum faciant utrum ligno an carboni an suo generi adnumerabimus? et magis est, ut proprium genus habeatur.'

En el texto Ofilio afirma, pues, que la leña destinada a hacer carbón, no es carbón; así Ulpiano considera otra nueva inteligencia de la cuestión, ya que escribe que en ese caso, tampoco podría entonces ser considerada leña, porque el testador la destinó con el fin de elaborar carbón. Por tanto ese material no sería ni carbón ni leña, y ha de ser considerado una categoría diferente, como asimismo ocurre con los tizones y las maderas quemadas para que ardan sin humo.

La diferencia explícita que aparece en el fragmento entre leña y carbón, se puede relacionar con otra de las formas en que se presenta el concepto actual de biomasa (si lignum sit paratum ad carbones coquendas atque conficiendas) concretamente la mencionada en tercer lugar como, «transformación química o biológica de ciertas especies vegetales»; es decir como dice en el texto «leña dispuesta para arder y hacer carbón ${ }^{31}$ ». Y esto también se sustenta en la relación de los diferentes usos y transformaciones químicas de la madera, como los tizones, que sirven para mantener el rescoldo del fuego durante largo tiempo y para encender fuego más fácilmente, y como la madera quemada que posteriormente arde sin humo. No podemos ignorar que en este último caso se está clasificando una especie de combustible, '... ... et alia ligna cocta ...' , que en su proceso productor de energía no propaga gases o partículas que puedan considerarse molestas o no deseables. Los romanos y antes los griegos, ya conocían al menos tres procesos por los que se obtenía leña sin humo, uno de los cuales era el que obtenía la ligna cocta o coctilia. La necesidad de este tipo de leña sin humo -coctilia- provenía del procedimiento usual para calentar las casas mediante el uso de hogares y braseros que necesitan combustibles que produjeran la menor cantidad de humo posible; al conjunto de procesos para la obtención de ese tipo de combustible los griegos

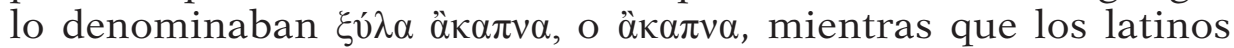
adoptaron el nombre por la cosa que se designa (acapna ligna). ${ }^{32}$

${ }^{31} \mathrm{Y}$ cierto es, que la leña sometida a un proceso de combustión sin oxígeno da lugar al llamado carbón vegetal.

${ }^{32}$ DAREMBERG-SAGLIO, Dictionnaire des Antiquités Grecques et Romaines, cit., voz Acapna, donde se refiere a acapna ligna como a la madera que se quema sin humo, usada en la antigüedad por los griegos y posteriormente por los romanos cuyas espacios geográficos presentaban climas templados que no requerían medios de calentar la domus muy complicados. En la Antigüedad, en sus casas disponían, como se hace actualmente en multitud de países cálidos, de hogares o braseros, y en el primer caso la evacuación de humo se hacía generalmente con una simple abertura en el techo, este modo de calentamiento hacía necesario el empleo de combustibles que produjeran la menor cantidad de humo 
Esta cuestión, no puede dudarse que sugiere realidades de actualidad $^{33}$.

Del mismo modo que sucede con los tizones y leña para quemar sin humo, también Ulpiano, en el mismo libro 25, escribe que no es leña sino clase aparte, alia, tanto las maderas sulfuradas -D. 32,55,8- como las teas -D. 32, 55,9- Así es, escribe el jurista que también la leña sulfurada se tendrá como género distinto -D.32,55,8-Sulpurata quoque de ligno aeque eandem habebunt definitionem y en relación a las teas tampoco han de comprenderse con el nombre de leña a no ser que fuera voluntad del testador, D. 32, 55,934 Ad faces quoque parata non erunt lignorum appellatione comprehensa, nisi haec fuit voluntas. Por el contrario las piñas de los pinos sí son leña D.32,55,10 $0^{35}$ De pinu autem integri strobili ligni apellatione continebuntur.

\section{II.4.A.c) Otras inclusiones al concepto de leña.}

El jurista en varios textos identifica qué puede considerarse como leña en algunas de las provincias romanas. Y es que, quizá, como consecuencia del hecho de la anexión progresiva de nuevas pro-

posible. Se servían de maderas que habían tenido la precaución de secar completamente antes de su utilización. Los griegos perfeccionaron los procedimientos de desecación hasta tal punto que obtuvieron maderas que quemaban sin producir ningún humo, y que deno-

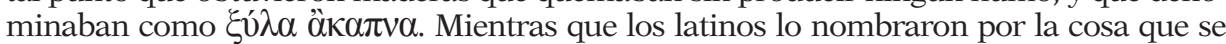
designa -acapna ligna-. Actualmente se conocen tres procedimientos de los que utilizaban, el más simple consistía en realizar la desecación de la madera exponiéndola a un fuego ardiente pero sin reducirla a carbón. La leña preparada de esta forma se llamaba también ligna cocta o coctilia (Martialis Epigrammae XIII,15). El segundo método consistía en eliminar la corteza e introducir la madera en agua y después proceder a su secado antes de su utilización (Theodo. Hist. Plant XV.10). El último procedimiento consistía en la inmersión en amurca, la parte acuosa de la aceituna que sale en el primer proceso antes de obtener el olea (Cato, De Re Rustica 130, y Plinio Hist. Nat. XV.8).

${ }^{33}$ Aún hoy en día, en determinados espacios geográficos se utiliza y recomienda esta técnica primero griega y posteriormente romana. El Instituto de Tecnología del Agua de México, que depende de la Secretaría de Medio Ambiente y Recursos Naturales del Gobierno Federal -SEMARNAT-, ha publicado en fechas recientes un «Manual del fogón sin humo" para las familias que cocinan aún con leña con el fin de ayudar a la población a mejorar las estufas y cocinas utilizando combustibles de biomasa en regiones en desarrollo, utilizando las técnicas de leña sin humo. El objetivo principal de la propuesta es contribuir en lo inmediato al mejoramiento de las condiciones de vida de las familias rurales que cocinan con leña, buscando eliminar la contaminación del interior de las viviendas por el humo y de manera importante disminuir el consumo y el corte de árboles. El uso del fógón sin humo es más eficiente, contamina menos el entorno, y en el mediano plazo contribuye a la conservación de los montes o bosques, suelo y agua.

${ }^{34}$ Momm. ref. nt.1 que señala el texto como geminado con D. 50,16,167.

${ }^{35}$ Momm. ref. nt.1 que señala el texto como geminado con D. 50,16,167. 
vincias al Imperio, este jurista aprecia la necesidad de delimitar la noción para los nuevos hábitats de los territorios incorporados y de referir qué puede o no englobarse con el término «leña».

Ulpiano, D.32,55,5, en relación a algunas regiones, como Egipto, escribe que con el nombre de leña entran también las cañas, y de este modo pueden quemarse tanto las cañas y las plantas de papiro, algunas yerbas, los espinos y abrojos ${ }^{36}$.

'Lignorum appellatione in quibusdam regionibus, ut in Aegypto, ubi harundine pro ligno utuntur, et harundines et papyrum comburitur ${ }^{37}$ et herbulae quaedam vel spinae vel vepres continebuntur. quid mirum? ...'

En sentido similar al fragmento anterior Ulp.25 ad Sab. D.32,55,6 escribe que en algunas provincias usan también para quemar el estiércol de los bueyes, In quibusdam provinciis et editu bubum ad hanc rem utuntur. Por el contrario negando el carácter de leña cabe citar de nuevo a los frutos del quercus, la bellota, de la que ya se ha señalado supra que se producía especialmente en Hispania.

Ulpiano, amplía el margen del concepto de leña, lignum, como combustible, a los residuos orgánicos mezclados cuando transcribe que el estiércol de los bueyes también se utiliza en algunas provincias $^{38}$. Con ello, y extrapolando a la terminología de nuestros días con la que se inicia el presente estudio puede observarse que residuos como el estiércol se incluyen expresamente como forma de biocombustible.

En definitiva, este jurista utiliza con carácter general un sentido lato del término lignum, que en la relación jurídica de legado testamentario de leña, favorecería al legatario, en detrimento del heredero, pero siempre que esos recursos sean expresamente dispuestos para quemar, esto es, no en los casos que sirva para otras utilidades concretas, a excepción de que sea ésta la voluntad del dueño o testador.

${ }^{36}$ LEVY-RAVEL, Index Interpolationum, sobre el tenor de este fragmento recaen diversas sospechas de interpolación del fragmento original. Schulz considera como interpolado el texto entre harundines y comburitur. Seckel desde papyrum a vepres., Lenel desde et papyrum a comburitur. y en la Palingenesia de Lenel, está ubicado bajo el título XXV De legatis, rúbrica De ligno legato, col 1.106, n² 2679, cfr. LENEL, O., Palingenesia Iuris Civilis.

${ }^{37}$ Momm. ref nt.24 comburitur.

${ }^{38}$ D'ORS et alii, El Digesto de Justiniano, cit., en la traducción utiliza el término «quemar» dado que ha de sobreeentenderse ya que en el texto original no figura. 
II.4.A.d) La leña en el legado de un fundo.

La jurisprudencia trata del concepto de leña, no sólo a efectos de la discusión de qué ha de entenderse por la misma en el legado de leña, sino también en un caso diverso, a propósito de la venta o legado de un fundo. En este último supuesto la decisión de Ulpiano, Ulp. 32 ad ed, D.19,1,17,2 sobre quién ha de quedarse con la leña es la que sigue ${ }^{39}$ :

Fundo vendito vel legato sterculinum et stramenta emptoris ${ }^{40}$ et legatarii sunt, ligna autem venditoris vel heredis, quia non sunt fundi, tametsi ad eam rem comparata sunt. in sterculino autem distinctio Trebatii probanda est, ut, si quidem stercorandi agri causa comparatum sit, emptorem sequatur, si vendendi, venditorem, nisi si aliud actum est: nec interest, in stabulo iaceat an acervus sit.

Comentando el jurista al edicto del Pretor, dispone que en el caso de que lo legado -o vendido- sea un fundo, el estercolero y la paja son del legatario -o comprador-, pero la leña del fundo será para el heredero -o vendedor-, porque no es del fundo, pero se compró para él. En este caso, interpretamos, Ulpiano no está considerando el estiércol como leña, por el criterio de la propia finalidad. El hecho de que determinados recursos se destinen a leña, implica la exclusión del resto en tal categoría. Vemos, una vez más, como opera en Ulpiano este criterio de que será leña cuando así se hubiera previsto específicamente. El jurista recurre a la distinción de Trebacio, en el sentido de que si verdaderamente hubiera sido comprado para estercolar el campo, sigue al comprador, y si para venderlo, al vendedor, si no se trató otra cosa; y no importa, como comenta el jurista que esté extendido en el establo, o que se haya dispuesto en montón.

\section{II.4.B. Pomponio y Paulo}

Pomponio y Paulo tratando de un legado de madera han describir lo que ha de comprenderse como materia, pero también como lignum, porque recordamos a este punto cómo aquello que ha de integrar un legado de madera hay que interpretarlo a la luz de lo

${ }^{39}$ LENEL, Palingenesia Iuris Civilis, col. 655, n 938, bajo la rúbrica De actione venditi, y el título Additamenta.

${ }^{40}$ Momm, ref. nt. 12, vel. 
concebido por leña. Y así lo hace Paulo, Paul 4 ad Sab. D. 32, 56 tras diferenciar entre leña y madera considera como materia los palos y las pértigas. ${ }^{41}$ Pali et perticae in numero ${ }^{42}$ materiae redigendi sunt, et ideo lignorum appellatione non continentur ${ }^{43}$. Anteriormente Pomponio, Pomp. 30 ad Sab. D. 32,57 citando a Servio y éste a Sabino también describe qué ha de considerarse como madera -arca y armario-Servius respondit, cui omnis materia legata sit, eic nec arcam nec armarium legatam esse.

\section{II.5. Reelaboradores postclásicos y justinianeos. El Libro 50,16 de Digesto De verborum significatione}

Para completar este análisis, traemos a colación una constitución imperial del primer tercio del S. IV, en la que curiosamente en una cuestión ajena a la problemática estudiada se mantiene la diferencia expuesta entre madera, leña, y carbón:

C.10,48,12,3 Grat./ Valent./ Theod;. 3 Operarum atque artificum diversorum, excoquendae etiam calcis obsequia nulla de talibus adiumenta poscantur: materiam lignorum atque tabulata exceptorum virorum patrimonia non praebeant: carbonis quoque nisi eum, quem moneta sollemniter vel fabricatio secundum veterem morem poscit armorum, ab huiusmodi viris praebitio desistat: publicis vel sacris aedibus construendis atque reparandis, capituli atque temonis necessitas nulla mandetur ....

En el texto los emperadores disponen que no se pidan auxilios a los operarios ni determinados servicios, como la cocción de la cal, el suministro de leña, ni tablas, ni carbón, y que no se les imponga ninguna obligación de suministrar capitel y traviesa para construir y reparar edificios públicos o sacros. Dada a 5 de los Idus de Diciembre, bajo el Consulado de Antonio y de Siagrio.» De la lectura de este texto se advierte la mención con carácter excluyente, de «leña» y «tablas»-para madera-. Por ello reiteramos la continuidad de este criterio en la etapa postclásica romana. Por otra parte, la postura que mantiene la diferencia de los materiales leña y carbón es la que asimismo perdura en este periodo y así lo recogen los emperadores Graciano, Valentiniano y Teodosio.

${ }^{41}$ Momm. ref. nt. 2, indica D.50,16,168 como texto geminado.

${ }^{42}$ Momm. ref.nt. 3 indica lege geminata pero con la diferencia del término $n u$ merum.

${ }^{43}$ LEVY-RAVEL, Index Interpolationum, Beseler entiende que desde et ideo al fin del fragmento no es original. 
Por último, cerrando el arco histórico que hemos pretendido trazar, la expuesta opinio de Ulpiano, 25 ad Sab. sobre la noción de leña, y su diferencia con la madera, carbones y otros, se dispone por el compilador en el Libro 50,16 de Digesto, De verborum significatione ${ }^{44}$. Este es el objeto de la Similitud n ${ }^{\circ} 5.165$, con la que se iniciaba este estudio jurisprudencial, y que se compone de dos textos. El texto-A- es de Ulpiano, Ulp. ad Sab., D.32,55,7. En la Palingenesia de Lenel ${ }^{45}$, está situado bajo el título XXV De legatis, en particular, De ligno legato, y bajo la columna $1106 \mathrm{n}^{\circ} 2679$. Ubicado en Digesto en el libro 32 sobre los legados y fideicomisos, la doctrina ha detectado posibles interpolaciones $^{46}$. En cuanto al texto -B- su inscriptio es también Ulpiano 25 ad Sab., D.50,16,167, con orden palingenético ${ }^{47}$, columna 1106, $\mathrm{n}^{\circ}$ 2679, título XXV De legatis, en particular, De ligno legato. Se ubica en Digesto en el libro 50,16,167 bajo el título De verborum significatione, y la doctrina no lo ha considerado interpolado ${ }^{48}$. El texto objeto de similitud se marca en letra negrita. Según lo dispuesto en la doctrina sobre coincidencias textuales en Digesto aquellos que pertenecen a un mismo autor, obra y libro han de ser considerados geminaciones ${ }^{49}$.

${ }^{44}$ Sin perjuicio de que ya se haya explicado supra, cabe reiterar que en D.50,16 los compiladores manifestaron mediante una selección de términos la importancia de las palabras y de su significado jurídico. Autores que han tratado específicamente de este título de Digesto son BIONDI, B., Valore delle etimologie dei giuristi romani, en Synteleia Arangio Ruiz, Napoli 1964. DELL'ORO, A., «Il linguaggio dei compilatori del Digesto quale risulta dal titolo 50.16 «De verborum significatione», Scientia iuris e linguaggio nel sistema giuridico romano, Milano 2001; MARRONE, M., «Le significationes di D. 50,16 ("De verborum significatione")», en SDHI 60 (1994); SCHMIDLIN, Die römischen Rechtregeln, Köl-Wien 1970; REINOSO-BARBERO, F., 'Definitio periculosa': ¿Iavoleno o Labeon?, en BIDR 90(1987).

${ }^{45}$ LENEL, O., Palingenesia Iuris Civilis, cit.

${ }^{46}$ LEVY-RAVEL, Index Interpolationum, en el que se cita como Beseler sugiere como interpolado el tenor entre sed-fin. Además Lenel sospecha de los términos carbonum et lignorum.

${ }^{47}$ LENEL, O., Palingenesia Iuris Civilis, cit.

${ }^{48}$ LEVY-RAVEL, Index Interpolationum, donde no figura como interpolado este texto.

${ }^{49}$ Las geminaciones son textos de Digesto con redacciones iguales, para conocer las diversas teorías vid. GARCÍA GARRIDO, M.J. Redacciones coincidentes (Leges geminatae) y casos jurisprudenciales semejantes (capita similia), cit. pág. 517 y ss.; Reiteramos nuevamente en esta sede la obra de REINOSO BARBERO, F., Siete prevenciones en la interpretación del lenguaje jurisprudencial, cit. Además de la bibliografía que se cita supra en la que no sólo se trata de similitudes sino también de geminaciones, puede consultarse BLUHME, De geminatis et similibus queae in Digestis invenientur capitis, Jena 1820; CHIAZZESE, Confronti testuali. Contributo alla dottrina delle interporlazioni giustinianee, cit. pág. 65 y ss; MARTÍN MINGUIJÓN, A., I testi geminati e loro confronto con la Massentheorie Bluhmiana,-en prensa-Archivio Giuridico, "Filippo Serafini», ed. Mucchi, Modena; REINOSO BARBERO, F., Geminaciones ocultas en el Digesto, en Index 25 (1997) 207 y ss.; VERREY, Leges geminatae à deux auteurs et compilation du Digeste, Laussane 1973. 


\section{Similitud $\mathrm{n}^{\circ} 5.165$}

TEXTO A

\section{D.32,55,7 Ulp. 25 ad Sabinum ${ }^{50}$}

'Si lignum sit paratum ad carbones coquendas atque conficiendas, ait Ofilius libro quinto iuris partiti carbonum appellatione huiusmodi materiam non contineri: sed an lignorum? et fortassis quis dicet nec lignorum: non enim lignorum gratia haec testator habuit. sed et titiones et alia ligna cocta ne fumum faciant utrum ligno an carboni an suo generi adnumerabimus? et magis est, ut proprium genus habeatur.'
TEXTO B

D.50,16,167 Ulp. 25 ad Sabinum

"Carbonum»appellatione materiam $^{51}$ non contineri: sed an "lignorum?" et fortassis quis dicet nec lignorum: non enim lignorum gratia ${ }^{52}$ habuit. sed et titiones et alia ligna cocta ne fumum faciant utrum ligno an carboni an suo generi adnumerabimus? et magis est, ut proprium genus habeatur. sulpurata quoque de ligno aeque eandem habebunt definitionem. ad faces quoque parata non erunt lignorum appellatione comprehen$s a$, nisi haec fuit voluntas. idem et de nucleis olivarum, sed et de balanis est, vel si qui alii nuclei $i^{53}$. de pinu autem integri strobili ligni appellatione continebuntur.'

En principio habría que presumir que la coincidencia entre ambos textos presenta el mismo tenor literal y el mismo contenido, y ello por la razón básica de que pertenecen ambos fragmentos al mismo autor, libro y obra, pero además también puede entenderse que el compilador tomó el texto de Ulpiano y lo dispuso en D.50.16 para explicar el término de leña sin manipularlo -ya que como se ha mencionado D.50,16,167 no se considera interpolado por la doctrina-. Sin embargo por nuestra parte observamos algunas diferencias en su literalidad y en la interpretación del concepto, sobre las cuales sugerimos, en virtud de los estudios de la doctrina sobre textos se-

${ }^{50}$ Momm. ref. nt. 28, los fragmentos de D. 32, 55, 7 a 9 se encuentran repeticiones en D. $50,16,167$.

${ }^{51}$ Momm. ref. nt. 5 y que remite como ley geminada al primer inciso del texto contenido en D. 32, 55,7.

52 Momm. ref.a nt. 6 y que remite como ley geminada a D. 32, 55,7.

${ }^{53}$ Hace referencia a la nota 7 que figura en el texto de Mommsen, y que remite como ley geminada a D. 32, 55,7. 
mejantes, que pueden deberse a que el compilador manejó diversas versiones del texto de un mismo jurista ${ }^{54}$.

Examinamos las concomitancias y diferencias de tenor y contenido, primero, en referencia sólo a la parte que presenta similitud, y segundo, entre los dos pasajes íntegros.

Semejanzas y diferencias en el texto objeto de la Similitud:

La concomitancia literal se concreta en el siguiente tenor: "Carbonum»appellatione materiam ${ }^{55}$ non contineri: sed an "lignorum?» et fortassis quis dicet nec lignorum: non enim lignorum gratia ${ }^{56}$ habuit. sed et titiones et alia ligna cocta ne fumum faciant utrum ligno an carboni an suo generi adnumerabimus? et magis est, ut proprium genus habeatur». La parte pues que coincide en ambos textos se traduciría en el sentido de que si hay leña dispuesta para arder y hacer carbón, ésta no ha de comprenderse en el nombre de carbones, y ante la pregunta de Ulpiano sobre si entonces ha de considerarse leña i.... sed an lignorum? se responde que cabe considerarlo como clase aparte. Se cuestiona también si los tizones y otra leña quemadas para que ardan sin humo han de contarse como leña o como una clase aparte para finalmente considerar en el texto que es mejor tenerlo como clase aparte.

En relación con las divergencias en la similitud, se señalan con negrita las diferencias de tenor literal: carbonum appellatione huiusmodi materiam non contineri: sed an lignorum? et fortassis quis dicet nec lignorum: non enim lignorum gratia haec testator habuit. En D.32,55,7 aparecen dos expresiones o vocablos haec testator y huismodi, que sin embargo se omiten en D. 50,16,167. La expresión haec testator que aparece en el texto -A- puede deberse a que manipulaciones posteriores de los textos hayan contemplado la coherencia de estar situado en el libro correspondiente a los legados como disposiciones testamentarias o bien ubicarse en el libro sobre el significado de las palabras de D.50,16 donde habría carecido de sentido encontrar tal expresión. La inclusión del término huismodi cuando se cita a Ofilio en D.32,55,7 puede dar lugar a una diversa interpretación.

En atención al contenido, hay que tener en cuenta que la cita a Ofilio que figura en D.32,55 se omite en D.50,16,167 lo que puede implicar una diversa comprensión de éste último. Así pues, te-

${ }^{54}$ Vid. sobre los diversos tipos de similitudes la doctrina mencionada supra.

55 Momm. ref. nt. 5 y que remite como ley geminada al primer inciso del texto contenido en D. 32, 55,7.

56 Momm. ref.a nt. 6 y que remite como ley geminada a D. 32, 55,7. 
niendo esto en cuenta, el texto objeto de similitud, interpretamos puede presentar dos lecturas diversas dependiendo de si lo insertamos en el texto A o en el texto B. En D.32,55,7, donde se cita a Ofilio, se inicia la explicación del concepto de leña-lignum- partiendo de la noción de leña y su diferencia con el carbón, mientras que en D.50,16,167 se expone el mismo concepto pero diferenciando entre madera -materiam- y el carbón. Y ello de modo que, mientras que en D.50,16,167 parece resultar evidente la distinción entre madera y carbón, la disparidad entre leña y madera no figura tan nítida.

En el contexto histórico actual español ésta cuestión está resuelta en términos estrictamente lingüísticos en el mismo sentido que los juristas clásicos, ya que el DRAE ${ }^{57}$ entiende por «Madera», en su primera acepción, la parte sólida de los árboles cubierta por la corteza y también el primer significado que nos ofrece de «Leña» es la parte de los árboles y matas que cortadas y hechas trozos se emplea para combustible. Pero en el haber de nuestro diccionario de la Real Academia aparece asimismo el vocablo «maderable» como designación de árboles o bosques utilizables para usos industriales -como construir, reparar y edificar- constituyendo una diferencia respecto a la concepción romana. Sin embargo, ponemos de relieve que, a pesar de la neta separación de estas voces en nuestro acervo lingüístico, aún en nuestros días para los ecólogos se plantea el dilema de si incluir o no la madera para estimar la biomasa de un bosque ${ }^{58}$.

Por otra parte, transcendiendo de la propia similitud y poniendo en relación los dos pasajes íntegros $-\mathrm{A}-\mathrm{y}-\mathrm{B}-$ cabe señalar lo que sigue. Respecto a la primera parte de D.50,16,167, en concreto desde «Carbonum apellatione ... hasta ... et magis est, ut proprium genus habeatur» puede decirse que en apariencia los compiladores al incluir el párrafo en el libro 50,16 definiendo la «madera», y también precisando la noción de leña, han unido algunos de los fragmentos incluidos en el comentario de Ulpiano a Sabino -D.32,55-. El conjunto que resulta de tal anexión es poco esclarecedor, puesto que mezcla sin mucho método explicaciones del significado de leña, de

57 Real Academia Española, Diccionario de la Lengua Española, cit. vol. 2.

58 En este sentido, MAGALEF, R., Ecología, ed. Omega, 1974; Sobre ecología consúltese también otros autores como.; CASADO, S., Historia de la Ciencia Ecológica en España, ed. Banco Santander, 2010; DE BUJÁN FERNÁNDEZ, F., La vida. Principio rector del Derecho, Madrid, ed. Dykinson, 1999; MOLLÉS, M. Ecología: Conceptos, aplicaciones, Madrid, ed. McGraw-Hill, 2006; STORER, J. La trama de la vida: introducción a la ecología, ed. Fondo de Cultura Económica, 1966. 
madera y de carbón. La apariencia es, en un principio, establecer el concepto de madera, como lo corrobora también el espíritu del fragmento D.50,16,168 donde se refiere que los palos y las pértigas deben contar como madera, y no entran por ello en la denominación de leña, Paulus libro quarto ad Sabinum, Pali et perticae in numerum materiae redigendi sunt, et ideo "lignorum» appellatione non continentur. Por exclusión también define la noción de leña. En realidad el texto parece simplemente pretender concentrar las tres especies que con mayor relevancia se discuten en el comentario de Ulpiano a Sabino, D.32,55.

Cabe decir asimismo y concluir, que por la relación entre los dos pasajes completos $-\mathrm{A}-\mathrm{y}-\mathrm{B}-$ se encontrarían comprendidos en D.50,16,167:

a) El objeto de la similitud examinada correspondiente a D.32,55,7 con los matices ya expuestos.

b) Literalmente, están incluidos en D.50,16,167, los siguientes fragmentos de D.32,55 a modo de geminaciones -y que Mommsen los detecta como leges geminatae-:

-D.32,55,8, como geminación. En la obra de Digestorum Similitudines, se detecta como similitud $\mathrm{n}^{\circ} 9.166$, pero que rigurosamente clasificada constituye una geminación, y que indica que las maderas sulfuradas no son leña.

-D.32,55,9 se recoge en la Similitud $n^{\circ} 273$, puede catalogarse como una geminación, mencionando que las teas no se comprenderán como lignum, salvo voluntas testatoris.

-D.32,55,10 corresponde a la Similitud n ${ }^{\circ} 1.261$, también asimismo geminación en relación a las piñas enteras de los pinos.

c) Sin embargo, el texto comprendido en D.50,16,167 «idem et de nucleis olivarum, sed et de balanis est, vel si qui alii nuclei» estaría relacionado con D.32,55,1 -ya analizado a propósito de la opinio de Ofilio comentado por Ulpiano Ulp.25 ad Sap-. Mommsen lo detecta como lex geminata en nt. 5 al texto. Pero, si bien, existe una coincidencia textual parcial, ésta presenta un significado bien distinto en cuanto a si ha de considerarse las balani-bellotas-, y los huesos de aceituna -nuclei olivarum- objeto de leña. Así en el libro 32 de Digesto se entienden como incluidas en el legado de leña, sin embargo en el libro 50, la forma de colocar este texto hace pensar que han de excluirse de esta noción. 


\section{TRADICIÓN JURISPRUDENCIAL Y OTRAS CONCLUSIO- NES}

(I) La tradición jurisprudencial sobre el concepto de leña -lignumsería la constituida por los siguientes juristas, Mucio Scevola, Servio, Ofilio, Trebacio, Sabino, Pomponio, Ulpiano, Paulo, constituciones imperiales de Graciano, Valentiniano, Teodosio, y los compiladores -cuando disponen el texto de Ulpiano en el título De verborum significatione - utilizando, quizá, otra versión del mismo texto del jurista.

(II) En particular desde Mucio Scevola hasta Ulpiano parece mantenerse un criterio común de la noción, precisándose mediante la citada tradición jurisprudencial qué se entiende como tal. Consideran como leña, los árboles cortados y no cortados expresamente dispuestos para ser partidos y quemar; también lo que no recibe otro nombre y sólo se puede aprovechar para hacer fuego; los árboles abatidos; y lo que se dispone específicamente para quemar, que en el caso de legado de leña correspondería a la voluntas testatoris.

En la jurisprudencia clásico tardía, con Ulpiano, se consideró específicamente que no es leña un bosque en su conjunto, el carbón, y la leña para hacer carbón, así como otros recursos que pudiendo básicamente entrar algunos en el concepto de leña por sus condiciones, sin embargo quedan reservados para otras utilidades.

En todas las fuentes clásicas destaca el hecho de que la adscripción al concepto de leña implica la designación expresa para ese fin, de forma que no se puede entender que es leña un bosque completo, será el árbol o árboles destinados para ello o los abatidos en su caso. Esta tendencia parece variar en la versión que de la respuesta de Ulpiano se incluye en D. 50, 16, 167.

(III) La noción romana de leña como se ha visto en el discurrir de los textos si la extrapolamos a nuestros días podría equipararse al concepto de biocombustible que se expuso inicialmente por dos razones. La primera, por similitud del fin 'comburo',- para producir la energía necesaria que precisen las distintas aplicaciones, -algunas de ellas se han mantenido en el devenir de la historia, como el uso de ésta para calefacciones, hornos, etc- ${ }^{59}$. La segunda por los materiales objeto de 'comburo', a saber los «combustibles», pues en

59 Coincide con el concepto que propongo, la opinión de d'Ors cuando calificó a la leña como combustible a propósito de tratar de los Bronces de Vipasca, vid. d'ORS, A., Epigrafía Jurídica de la España Romana, Madrid, Instituto Nacional de Estudios Jurídicos, 1953. 
la dilucidación de los textos hemos señalado distintos componentes que se incluían en el nombre de 'lignus', y que se ordenan y encajan fácilmente con las distintas formulaciones que aquí se daban de biocombustible o biomasa ${ }^{60}$.

(IV) El 'lignus', o leña y su posible disparidad con la 'materia' o madera, está basada en las fuentes jurídicas romanas en el libre arbitrio del titular del 'fundum', que realizará la adscripción de los materiales a una u otra opción según el contexto en que encuentre con esa relación de propiedad: propietario de un fundo, propietario de un fundo que compra la leña aparte, propietario testador, heredero testamentario, legatario. Es decir, está perfectamente determinado en el vocablo madera cuál es el elemento material que lo compone, diferenciándose claramente frente a la leña, y sin embargo se ha mantenido en el paso de los siglos la adscripción por el criterio de la finalidad.

Hoy en día existe un gran número de normas que tienen como objeto la protección de los bosques ante fines como ser utilizados para comercio de maderas -maderables- o como posible objeto de combustible, y que tienen el objeto de protección ecológica de los bosques $^{61}$.

${ }^{60}$ Diferenciando por el tipo de proceso del que se obtiene la energía, señalamos que los procesos que se llevaron a cabo en Roma que resultan similares a los actuales fueron, primero, la obtención de energía de biomasa derivada de residuos forestales, y segundo, la obtención de energía de la combustión de a través de la transformación química o biológica de ciertas especies vegetales en carbón. En el primer caso, con origen en la combustión de residuos agrícolas y domésticos podríamos englobar los residuos agrarios que cita tanto Ofilio y Ulpiano, principalmente los restos de sarmientos, varas pequeñas, matorrales, virutas, troncos y raíces de vides. Además, en particular a juicio de Ulpiano también el estiércol, las plantas de papiro, espinos, abrojos y otras yerbas. En el segundo caso, todos los supuestos de conversión en carbón.

${ }^{61}$ Cabe citar, únicamente a título de mención por exceder del objeto de este trabajo, en el ámbito del Ordenamiento jurídico español la ley 42/2007 de 13 de diciembre, del Patrimonio Natural y de la Biodiversidad, que en su artículo 76 y sin perjuicio de lo que disponga al respecto la legislación autonómica, dispone una relación de conductas atentatorias contra las condiciones de espacios naturales. A nivel internacional, y haciendo particular referencia a la finalidad de la explotación de los bosques para el comercio maderero, y entre otros muchos instrumentos de interés, señalamos el Convenio Internacional de las Maderas Tropicales de 2006 que ha sido ratificado por parte del Estado Español y que prevé entre sus objetivos promover el comercio de maderas tropicales de bosques ordenados de forma sostenible y aprovechados de forma legal, así como promover la ordenación sostenible de los bosques productores. 